\title{
Bases conceptuales y metodológicas de la Encuesta Nacional de Salud II, México, 1994*
}

\author{
Grupo Coordinador y de Diseño Conceptual de la ENSA-II
}

\section{Grupo Coordinador \\ y de Diseño Conceptual de la ENSA-II. Bases conceptuales y metodológicas de la Encuesta Nacional de Salud II, México 1994. \\ Salud Publica Mex I 998;40:76-85.}

\section{Resumen}

Se describen las bases conceptuales y metodológicas de la Encuesta Nacional de Salud II (ENSA-II), que integra avances de la investigación multidisciplinaria en salud pública, tanto en el terreno conceptual como en el metodológico, que se han dado en nuestro país últimamente. Su diseño se concentró particularmente en las condiciones del acceso, la calidad y los costos de los servicios de atención a la salud, tanto a nivel ambulatorio como hospitalario. Se incluyen detalles de su marco conceptual, así como los aspectos relacionados con el procesamiento y análisis. La cobertura geográfica fue hecha para cinco regiones; se visitaron 12615 viviendas a escala nacional, y se recabó información sobre 6I 524 individuos. La tasa global de respuesta fue de $96.7 \%$, tanto para los hogares como para los utilizadores identificados de servicios de salud. La conclusión general apunta hacia la incorporación del enfoque de la población al proceso de planeación y asignación de recursos para la atención a la salud.

Palabras clave: accesibilidad a los servicios de salud; calidad de la atención de salud; cobertura de servcicios de salud; México

\author{
Group for Coordination \\ and Conceptual Design of the NHS-II. \\ Conceptual and methodological basis \\ of the National Health Survey II, \\ Mexico 1994.
}

Salud Publica Mex 1998;40:76-85.

\section{Abstract}

The conceptual and methodological basis of the National Health Survey II (NHS-II) are described and recent advances in multidisciplinary public health research in Mexico, both conceptual and methodological, are synthesized. The design of the NHS-II concentrated on the study of the access, quality of care and health attention expenses in ambulatory and hospitalization services. Details on the conceptual framework related with the analysis and processing of data are also included. Five geographic regions were covered; 12 615 households at national level were visited and information on 61524 individuals was gathered. The overall response rate was $96.7 \%$ both for households and for identified health service users. The general conclusion emphazises the need to incorporate the population perspective to the planning and allocation of health resources.

Key words: health services accesibility; quality of health care; health services coverage; Mexico

\footnotetext{
* Los miembros del Grupo Coordinador y de Diseño Conceptual de la ENSA-Il que participaron en la elaboración de este trabajo son: Patricia Nájera
} Aguilar, Mauricio Hernández Avila, Roberto Tapia Conyer, Miguel Angel Lezana, José Gómez de León, Gustavo Olaiz Martínez y Jaime Sepúlveda Amor. 


\section{La Encuesta Nacional de Salud II}

Los patrones de morbilidad y mortalidad en México atraviesan por un periodo de profundos cambios. Hace apenas unos años las principales causas de mortalidad eran las enfermedades infecciosas; hoy en día, las crónico-degenerativas y las neoplasias ocupan los primeros sitios. A este proceso se le ha denominado transición epidemiológica, y se ha caracterizado por la presencia de ambos tipos de enfermedades en un contexto social, demográfico y económico cambiante. ${ }^{1}$ Para resolver adecuadamente las necesidades de servicios de salud ocasionadas por la transición epidemiológica se requieren cambios sustanciales en el volumen, tipo y calidad de los recursos de atención a la salud que serán necesarios para hacer frente a los rezagos y satisfacer las nuevas demandas de atención de la población mexicana. ${ }^{2}$

En este marco, la Secretaría de Salud llevó a cabo la Encuesta Nacional de Salud II (ENSA-II), que es la continuación del esfuerzo desplegado para estructurar y consolidar el Sistema de Encuestas Nacionales de Salud con el propósito específico de identificar las distintas modalidades en las que se presenta el contacto entre la población y los servicios de salud.

En la ENSA-II se han abordado los temas más relevantes en salud; sin embargo, dado que los aspectos referentes a la morbilidad y las condiciones de salud de la población han sido objeto de estudio en encuestas previas, esta encuesta se concentró particularmente en las condiciones del acceso, la calidad y los costos en los servicios de atención a la salud.

Las experiencias de investigación previas, consideradas como base para el diseño de la ENSA-II, fueron los proyectos: “Cobertura y calidad de la atención

\footnotetext{
* Instituto Nacional de Salud Pública. Cobertura y calidad de la atención primaria a la salud, México-BID. Informe técnico. 1988. Documento no publicado.

‡ Instituto Nacional de Salud Pública. Accesibilidad y calidad. Efectos en la utilización y percepción de los servicios de salud. Consejo Asesor en Epidemiología-Fundación Rockefeller. Informe técnico.1991. Documento no publicado.

§ Secretaría de Salud,Centro de Estudios en Población y Salud. Utilización de servicios en el área de Chimalhuacán, Estado de México, 1993. Documento no publicado.

* Secretaría de Salud, DGEIE. Patrones de cobertura y utilización de servicios de salud en el Distrito Federal, 1993. Documento no publicado.

${ }^{f}$ Secretaría de Salud,Unidad Coordinadora Central del Programa Solidaridad. Estudio cualitativo-diagnóstico sobre demanda de servicios de salud en las entidades PASSPA, 1993. Documento no publicado.
}

primaria a la salud, México-BID”;; “Accesibilidad y calidad. Efectos en la utilización y percepción de los servicios de salud"; ‘ "Utilización de servicios en el área de Chimalhuacán, Estado de México"; "Patrones de cobertura y utilización de servicios de salud en el Distrito Federal"," y "Estudio cualitativo-diagnóstico sobre demanda de servicios de salud en las entidades PASSPA". $f$

Pese a que constituyen una aproximación al conocimiento de la interacción entre servicios y población, estos antecedentes se remitían a resultados en el ámbito local y carecían de datos que permitieran caracterizar, nacional y regionalmente, el proceso de búsqueda y obtención de la atención. Es por ello, que en este artículo se reseñan las bases conceptuales y metodológicas de la ENSA-II, así como los métodos para abordar los diversos temas de estudio correspondientes al panorama socioeconómico y de salud de la población encuestada; la utilización y la cobertura de los servicios formales de salud; los costos del proceso de búsqueda y obtención de la atención ambulatoria; la prescripción, el acceso y el gasto en medicamentos, y la percepción de la calidad de la atención en distintos servicios de salud en México. Los objetivos específicos de la ENSA-II fueron los siguientes:

1. Determinar y cuantificar la relación que existe entre los factores predisponentes y habilitadores (capacitantes) con la accesibilidad, la utilización, la cobertura y la calidad de los servicios de salud.

- Distinguir, entre los atributos demográficos y socioeconómicos de la población, aquellas características que se traducen en capacidad o poder de utilización.

- Analizar los factores económicos, geográficos y organizacionales que intervienen en la utilización de servicios. En especial se busca dimensionar los gastos directos e indirectos que influyen en la conformación de la demanda de servicios de salud.

- Estimar las coberturas de las instituciones públicas y privadas de atención a la salud en los ámbitos regional y nacional.

- Analizar los factores que inciden en la percepción de la calidad de la atención por parte de los usuarios.

2. Identificar el tipo de establecimientos que utiliza la población en distintas regiones de México.

- Conocer el grado de calidad otorgada por los servicios de salud, con base en indicadores selectos de estructura, proceso y resultado de la atención.

- Determinar la magnitud y las características de la utilización de servicios del sector privado por parte de la población mexicana. 


\section{Métodos}

\section{Diseño de la encuesta}

Para fines de estudio se dividió al país en cinco regiones, y cada una se definió como un estrato, lo cual permite elaborar inferencias a escala regional. La regionalización utilizada para la ENSA-II se puede observar en la figura 1. En las cinco regiones se seleccionó igual número de hogares.

La encuesta consta de dos cuestionarios principales, uno dirigido a todos los integrantes del hogar seleccionado y otro a las personas que notificaron haber hecho uso de los servicios de salud, en los quince días previos al levantamiento de la información.

\section{Unidades de observación y análisis}

En atención a los diferentes objetivos de esta encuesta, las unidades de análisis son:

1. La población de los hogares seleccionados, que se definió como todas aquellas personas que al momento de la encuesta residían en los hogares ubicados en cada región. Dicha población la constituyeron los miembros del hogar, nacionales o extranjeros, que residían habitualmente en viviendas particulares. Las viviendas institucionales no fueron objeto de la Encuesta. El informante sobre las características de la población de los hogares fue, en general, la mujer responsable del hogar, siguiendo, en orden de importancia, otras mujeres mayores de 18 años u hombres en el mismo grupo de edad.

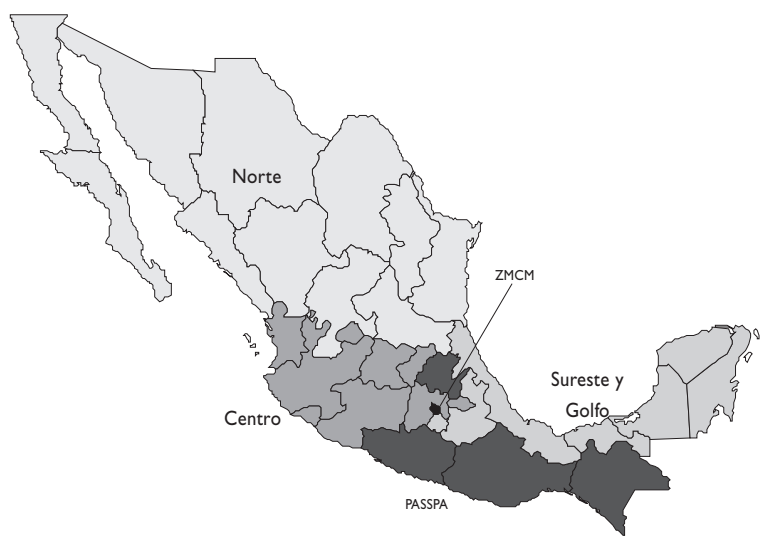

Figura I. Regiones de la Encuesta Nacional de SAlud II, México, 1994

salud pública de méxico / vol.40, no.1, enero-febrero de 1998
2. Durante el primer contacto con las viviendas seleccionadas se identificó a la población que utilizó algún servicio de salud en los 15 días previos a la entrevista. ${ }^{3}$ El informante de este cuestionario fue el utilizador, y en los casos en que éste era menor de 15 años, su madre o el (la) responsable del menor.

\section{Diseño de la muestra}

Para el diseño muestral, las entidades federativas se agruparon en cinco regiones de estudio, y para cada región se seleccionó un número aproximado de 2523 viviendas que se calculó con base en estimadores de uso de servicios de $15 \%$, una confiabilidad de $95 \%$ y un poder de $80 \%$. Estas cifras se consideraron adecuadas para los recursos presupuestales destinados a esta investigación.

Para la selección de las viviendas, en cada región se realizó un muestreo polietápico estratificado, utilizando el marco muestral jurisdiccional de viviendas de la Dirección General de Epidemiología (DGE). * Con el fin de lograr una distribución adecuada de las viviendas por estado, la muestra asignada para cada región se distribuyó de manera proporcional al tamaño de la entidad federativa, fijando un mínimo muestral de 100 viviendas para los estados más pequeños.

\section{Bases conceptuales}

Se han estudiado diferentes modelos que ilustran la relación entre los factores que determinan la variabilidad en los niveles de uso/demanda de los servicios de salud. El abordaje para este estudio se funda en trabajos previos de diferentes autores que proponen la clasificación de los factores que influyen en la utilización de servicios de salud: necesidades de salud, factores predisponentes y habilitadores o capacitantes (cuadro I).

La satisfacción del usuario ha sido incorporada al análisis de la utilización de servicios en dos sentidos: como variable dependiente o independiente en el proceso de utilización de servicios de salud. En el primer caso, como un indicador de resultado de la interacción entre proveedores y usuarios y, en el segundo, como predisponente para utilizaciones subsecuentes. ${ }^{16}$

Otro punto importante, en el cual coinciden los autores, es que la utilización debe entenderse como la interacción entre dos conjuntos de características: las

* Dirección General de Epidemiología-Secretaría de Salud. Marco muestral jurisdiccional de viviendas, 1994. Documento de trabajo no publicado. 


\section{Cuadro I \\ Factores QUe determinan la utiLización de SERVICIOS de SALUd}

I. Necesidades

Representan una de las causas más inmediatas del uso de los servicios, misma que varía en función de los factores predisponentes y habilitadores. Puede medirse con diferentes indicadores como son la percepción del estado de salud, la frecuencia de dolor, número de síntomas, días de actividad perdidos, discapacidad, severidad percibida de un problema, etcétera. ${ }^{5}$

II. Factores predisponentes

Edad. Predisposición a que los grupos etáreos extremos usen más servicios en forma paralela a los incrementos en morbilidad y mortalidad. ${ }^{6}$ Sexo. Por las diferencias de morbilidad y los episodios no mórbidos de salud en edad reproductiva, la mujer tiende a ser una mayor usuaria de los servicios de salud. ${ }^{4}$

Tamaño de la familia. Actúa en dos sentidos. El primero, como promotor del uso de servicios en el caso de enfermedades infecciosas. ${ }^{7}$ El segundo, la subutilización de servicios preventivos entre familias grandes por los grandes costos de oportunidad que ello implica. ${ }^{8}$

Grupo étnico. Traducido a nuestro país como pertenencia a una etnia de origen indígena o extensible a minorías no étnicas. La disponibilidad irregular de fuentes de atención y condiciones socioeconómicas propias de estos grupos minoritarios (baja capacidad de compra) impiden un uso regular de servicios. ${ }^{5}$

Educación. A mayor nivel de escolaridad mayor conocimiento se tendrá de la disponibilidad de los servicios de atención a la salud, así como de los beneficios en el estado de salud.?

Ocupación. Por los perfiles de morbilidad y accidentes laborales. ${ }^{10}$

Clase social. Es identificada como factor predisponente y asociada al nivel de ingreso." Existen programas preferenciales de atención a la salud dirigidos a poblaciones socioeconómicamente deprimidas. ${ }^{2}$

Cultura. Se ha estudiado, sobre todo, la forma en que influye sobre la percepción de la necesidad. No obstante, se establecen diferentes niveles de influencia de los factores culturales en la utilización de servicios, a saber, en la definición y evaluación de los síntomas. El segundo nivel, en el ordenamiento de prioridades y problemas de los individuos y grupos a que pertenecen. El tercero, en la vulnerabilidad percibida ante el encuentro médico-paciente. ${ }^{13}$ Según el esquema secuencial, los factores culturales podrían ubicarse en el momento en que surge el deseo de atención.

III. Factores habilitadores o capacitantes

Ingreso. Fuertemente asociado y difícil de identificar su independencia respecto a las condiciones de salud y laborales; no obstante, es un indicador directamente asociado a la superación o no de los obstáculos financieros. ${ }^{14}$

Derechohabiencia, seguro médico. Los individuos con esta característica son financieramente más capaces de adquirir servicios de atención a la salud sin erogar directamente ninguna cantidad..$^{15}$

Fuente: referencia 4

de la población y las de los servicios. Donabedian ${ }^{17}$ compara los contactos entre ambos actores en función de su comportamiento, como parte de una cadena de acontecimientos que inician en el reconocimiento de una necesidad de salud y derivan en la utilización de servicios después de haber pasado por las etapas de decisiones, de búsqueda y de asumir un papel como paciente; lo anterior en contraparte al proceso diagnóstico y terapéutico por parte de los proveedores, del cual se espera, como resultante, un cambio en el estado de salud.

Por otra parte, Aday y Andersen complementan el concepto de utilización de servicios de salud, no sólo en sus determinantes, sino que incorporan y especifican las características que ubican las condiciones en las que se da el contacto directo entre estos dos actores. Se pretende responder a las preguntas ¿por qué?, ¿dónde? ¿cuándo? y ¿con quién? se efectúa el contacto, para profundizar en el conocimiento de la utilización según el tipo de servicio empleado y el lugar en donde se efectuó el encuentro, la frecuencia y el recurso humano que otorgó la atención.

\section{Definiciones operacionales}

Para el estudio de la utilización de servicios se plantearon dos indicadores: la utilización general en los quince días previos al levantamiento de la información 
-que representa principalmente la morbilidad aguda, así como el uso de servicios preventivos en el primer nivel de atención-y la hospitalización en el último año -que representa la utilización del segundo y tercer nivel de atención-. La información sobre utilización en los 15 días previos se obtuvo de manera directa del usuario o su responsable; los datos sobre hospitalización se obtuvieron indirectamente del informante del hogar, y se cuenta con datos referentes al motivo de hospitalización, percepción de gravedad, días de estancia hospitalaria y el gasto de bolsillo.

Para dimensionar la necesidad de atención, se incluyó la percepción del estado de salud, la morbilidad percibida y la percepción de la gravedad como indicadores de necesidad desde el punto de vista de la población. Adicionalmente existen indicadores sobre la presencia, tipo y duración de la incapacidad.

En relación con los factores predisponentes y habilitadores, se obtuvo información sobre la edad, el sexo y la escolaridad. Entre los atributos familiares se tomaron como indicadores de predisposición el uso de servicios, el tamaño de la familia, la ocupación del jefe del hogar y el nivel socioeconómico. Como factores habilitadores se identificaron aquellas condiciones de la población que producen cambios relativamente independientes en los niveles de utilización como son el ingreso y la condición de aseguramiento. Esta última se evaluó de manera indirecta con la siguientes preguntas: “¿Tiene (Nombre) derecho a servicios médicos de seguridad social de alguna o algunas instituciones? ¿Tiene alguna otra?"

Las variables relacionadas con la accesibilidad se operacionalizaron a partir de una tipificación de los obstáculos secuenciales a los que se enfrenta la población para utilizar los servicios de salud y de las capacidades correspondientes que se ponen en juego para superar dichos obstáculos. ${ }^{18}$ La información recolectada abarcó factores o variables geográficos originados en la ubicación de las fuentes de atención como son las distancias, tiempos y costos de viaje; los financieros, asociados a los gastos de bolsillo que la población afronta durante todo el proceso de búsqueda y obtención de la atención, como los monetarios y los tiempos invertidos, y por último, los obstáculos organizativos, originados al interior de los servicios, previos a la atención misma y que son producto de las combinaciones de estructura y organización de la atención como los tiempos invertidos en cada proceso.

La cobertura se midió en diferentes niveles: 1. Nominal o legal. Por el número de personas que reportaron estar afiliadas a cada institución, según las instituciones de salud y su contraparte en la población.
2. Institucional. Por el número de afiliados que reportan haber hecho uso de los servicios que les corresponden por derecho o prioridad.

3. Por el número de personas que usaron los servicios ante una necesidad.

La calidad de la atención se abordó desde la perspectiva de los usuarios y se caracterizó mediante indicadores de percepción de la estructura, del proceso y del resultado. Se identificó la percepción sobre las características de la estructura (infraestructura y recursos) del proceso (relación interpersonal, contenido, duración e información proporcionada) y del resultado (percibido en los cambios de su estado de salud).

\section{Instrumentos de recolección de información}

Para el levantamiento de la encuesta se contó con los siguientes instrumentos: un cuestionario de hogar y un cuestionario individual para usuarios de servicios de salud. El primero es el instrumento de recolección de datos para conocer las condiciones en que se lleva a cabo la utilización de los servicios de salud. La población a la que fue aplicado estuvo constituida por mujeres responsables del hogar, mismas que proporcionaron la información sobre las características demográficas, socioeconómicas, percepción del estado de salud en el último año, condición de aseguramiento, tenencia de seguros privados de los integrantes del hogar, así como las características de la vivienda y religión. En el primer contacto en el hogar se recabó información sobre los integrantes que utilizaron los servicios de salud durante los 15 días previos a la encuesta; posteriormente se programó una cita con el/los usuarios de servicios identificados y se indagaron, en detalle, las características de la utilización de los servicios en los últimos 15 días. Asimismo, se preguntó sobre la utilización de los servicios demandados previamente. El contenido general de los cuestionarios se presenta en el cuadro II.

\section{Organización del trabajo de campo}

El levantamiento de información se llevó a cabo en siete etapas; se elaboraron cuatro manuales: del capacitador, del coordinador central, del supervisor y del entrevistador, en los cuales se describen las funciones y tareas de responsabilidad de cada una de las personas participantes, así como las definiciones conceptuales y operativas para cada nivel de ejecución.

La función del coordinador central fue cubierta por personal de salud con un mínimo de cinco años 


\section{Cuadro II \\ RELACIÓN de VARIABLES Y REACTIVOS EN LA ENSA-II}

Variables

Reactivos incluidos en los cuestionarios

Determinantes poblacionales

Necesidad de atención:

- Percepción del estado de salud

2.07

- Morbilidad percibida

$3.01,3.02,3.10$

- Percepción de la gravedad

$3.03,3.11$

- Requerimiento de servicios de carácter preventivo

3.18

- Incapacidad

2.11

Factores predisponentes individuales:

- Edad

2.02

- Sexo

- Escolaridad

$2.12,2.13,2.14$

- Estado civil

2.15

Factores predisponentes familiares:

- Tamaño de la familia

$2.01,2.05,(2.09,2.10,2.15,2.16)$

- Ocupación

- Nivel socioeconómico

- Religión

- Grupo lingüístico (étnico)

2.08

Factores habilitadores:

- Ingreso

$2.23,2.24$

- Derechohabiencia 2.06

Accesibilidad:

- Obstáculos geográficos

- Obstáculos financieros

I.06 vs I.46, I.08 (más tiempos y costos del viaje)

- Obstáculos organizativos

Patrones de utilización ambulatoria

- Autoayuda

$3.04,3.05,3.12,3.13$

- Automedicación

$3.06,3.07,3.14,3.15$

- Carrera del enfermo

$\mathrm{I} .4 \mathrm{I}, \mathrm{I} .42, \mathrm{I} .43, \mathrm{I} .44$

- Tradicionales y formales por:

Motivo

$1.01,1.02$

Frecuencia

1.45

Lugar (nivel y tipo de establecimiento)

Recurso (humano)

- Razones de no uso

1.03 (nivel), I.05 (tipo de establecimiento)

1.04

$3.09,3.17$

Hospitalización

- Motivo

- Frecuencia

- Lugar (nivel y tipo de establecimiento)

$4.01,4.02$

- Recurso (humano)

- Sistema de referencia

$4.06,4.07$

Accesibilidad:

- Obstáculos financieros

$4.08,4.09,4.10,4.11$

\section{Cobertura}

Nominal. Por el número de afiliados a cada institución Potencial. Por la capacidad de atender a la población afiliada Institucional. Por el número de afiliados que usan los servicios que les corresponden por derecho o prioridad

Real. Por el número de personal que usan los servicios de salud según sus necesidades de salud
$2.25,2.26$

3.18 en los servicios según recursos

$3.18,3.20,3.21$

Utilización/necesidades

(CONTINÚA) 


\section{Cuadro II \\ (Continuación)}

Variables

Reactivos incluidos en los cuestionarios

Calidad de la atención desde la perspectiva del usuario

Estructura:

- Infraestructura y recursos

I.II, I.18 (1.39, I.40)

Del proceso:

- Relación interpersonal I.15, a I.16

Contenido $1.17,1.23,1.29$

Privacidad

Duración

Información

1.17

I. 14

I. $17,1.24$

- Resultado (percibido en su salud)

1.36

- Satisfacción del usuario y peso atribuible a cada dimensión de la calidad

$1.37,1.38,1.39,1.40$

de experiencia en la supervisión de encuestas. El programa de capacitación se enfocó básicamente a los contenidos conceptuales de la encuesta, así como a la planeación de la capacitación a supervisores y entrevistadores en cada estado.

El trabajo de campo se organizó de manera independiente para cada estado y se inició en la región Norte, a la que siguieron la región Centro, la SuresteGolfo, grupos de estados incluidos en la primera fase del Programa de Apoyo en Solidaridad en Servicios de Salud para Población Abierta (PASSPA), que fueron Guerrero, Oaxaca, Chiapas e Hidalgo; y finalmente, la Zona Metropolitana de la Ciudad de México (ZMCM). Para cada estado se nombraron coordinadores centrales, dependiendo del acceso geográfico y la dispersión de la muestra. Con excepción de la ZMCM el trabajo de campo no excedió las tres semanas en cada región.

La coordinación de actividades en cada estado consintió en organizar reuniones informativas con las autoridades estatales de salud y los responsables del levantamiento de la DGE. El resultado básico de esa reunión fue la definición de los corresponsables operativos en el ámbito estatal.

Las autoridades estatales, con ayuda del coordinador central y en un lapso de dos semanas, reclutaron al personal de campo a partir de una convocatoria emitida a nivel jurisdiccional. La evaluación de candidatos se realizó de acuerdo con los perfiles predefinidos para cada función y a través de entrevistas de aptitudes para el puesto. Los seleccionados acudieron a un curso de capacitación que tuvo una duración de 40 horas.

\section{Procesamiento de la información}

Se realizó un procedimiento específico para el control cuantitativo de cuestionarios con un registro único in- tegrando las claves de la región, el estado, el municipio, la unidad primaria de muestreo, el área Geoestadística Básica (AGEB), el número de conglomerado, la vivienda, el hogar y la identificación de informante e integrantes del hogar. La estructuración de las bases de datos se efectuó a través de los procedimientos habituales de captura y de la aplicación de programas específicos de validación y criterios de congruencia. Los intervalos de confianza de las varibles principales se estimaron con el paquete SUDAAN. ${ }^{19}$ El análisis estadístico se realizó con en el paquete estadístico SPSS 4.0. ${ }^{20}$ La limpieza de datos se hizo por medio de los procedimientos denominados examine y leaf del mismo paquete. La construcción de escalas o índices se efectuó, con base en los resultados de encuestas previas y por consenso de expertos, en reuniones de trabajo ex-profeso para la preparación del informe técnico.

\section{Resultados del levantamiento}

En total se visitaron 12615 viviendas en el país, lo que significó recabar información sobre 61524 individuos. La tasa global de respuesta fue de $96.7 \%$, tanto para las vivendas como para los utilizadores de servicios de salud que fueron identificados. El número de hogares entrevistados a nivel nacional se presenta en el cuadro III. Un hogar fue definido con base en la declaración de compartir gastos para comer entre individuos residentes en una vivienda.

La distribución de las principales variables dan cuenta del tipo de población entrevistada (cuadro IV). En principio, $68.33 \%$ de la población entrevistada residía en comunidades urbanas al tiempo del levantamiento. Por otra parte, $49.45 \%$ correspondió a individuos de sexo masculino. La pirámide de edades de la población entrevistada, fue muy similar a la obtenida 


\section{Cuadro III}

Hogares Visitados para la

Encuesta Nacional de Salud II. México, 1994

\begin{tabular}{|c|c|c|}
\hline Región & Entidad & Hogares visitados* \\
\hline Norte & $\begin{array}{l}\text { Baja California } \\
\text { Baja California Sur } \\
\text { Sonora } \\
\text { Chihuahua } \\
\text { Coahuila } \\
\text { Nuevo León } \\
\text { Tamaulipas } \\
\text { Sinaloa } \\
\text { Durango } \\
\text { Zacatecas }\end{array}$ & 2510 \\
\hline Centro & $\begin{array}{l}\text { San Luis Potosí } \\
\text { Guanajuato } \\
\text { Querétaro } \\
\text { Michoacán } \\
\text { México } \\
\text { Tlaxcala } \\
\text { Nayarit } \\
\text { Aguascalientes } \\
\text { Jalisco } \\
\text { Colima }\end{array}$ & 2520 \\
\hline $\begin{array}{l}\text { Zona Metropolitana } \\
\text { de la Ciudad de México }\end{array}$ & Distrito Federal & 2520 \\
\hline PASSPA & $\begin{array}{l}\text { Hidalgo } \\
\text { Oaxaca } \\
\text { Guerrero } \\
\text { Chiapas }\end{array}$ & 2515 \\
\hline Sureste-Golfo & $\begin{array}{l}\text { Morelos } \\
\text { Puebla } \\
\text { Veracruz } \\
\text { Campeche } \\
\text { Tabasco } \\
\text { Yucatán } \\
\text { Quintana Roo }\end{array}$ & 2550 \\
\hline
\end{tabular}

Tota

12615

* Un hogar corresponde al conjunto de personas que comparte un mismo gasto para comer al interior de una vivienda

a partir del Censo Nacional de Población y Vivienda de $1990 .^{21}$

\section{Conclusiones y perspectivas}

Esta encuesta constituye una valiosa veta que busca complementar las fuentes regulares de información generada en los sistemas del Sector Salud.

\section{Cuadro IV}

Comparación de LAS fRecuencias Simples E INTERVALOS DE CONFIANZA AL 95\% DE LAS PRINCIPALES VARIABLES DE LA ENCUESTA CON LOS PAQUETES ESTADÍSTICOS

Variables

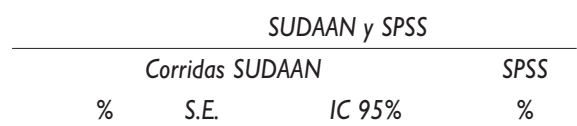

Tipo de comunidad

\begin{tabular}{lllll} 
Rural & 31.669 & 0.035 & $31.600-31.738$ & 31.7 \\
\hline Urbana & 68.331 & 0.035 & $68.262-68.400$ & 68.3 \\
$\begin{array}{l}\text { Sexo } \\
\text { Hombre }\end{array}$ & 49.453 & 0.220 & $49.022-49.884$ & 49.5 \\
\hline Mujer & 50.547 & 0.220 & $50.116-50.978$ & 50.5
\end{tabular}

Condición de aseguramiento

\begin{tabular}{lllll} 
Asegurado & 40.455 & 0.177 & $40.108-40.802$ & 40.5 \\
\hline No asegurado & 59.545 & 0.177 & $59.198-59.892$ & 59.5
\end{tabular}

Tipo de aseguramiento

\begin{tabular}{lrrcc} 
IMSS & 30.453 & 0.169 & $30.122-30.784$ & 30.5 \\
\hline No asegurados & 59.468 & 0.177 & $59.121-59.815$ & 59.5 \\
\hline ISSSTE & 6.964 & 0.097 & $6.774-7.154$ & 7.0 \\
\hline Pemex & 0.671 & 0.029 & $0.614-0.728$ & 0.7 \\
\hline Defensa-Marina & 0.418 & 0.025 & $0.369-0.467$ & 0.4 \\
\hline Otra inst. pública & 1.244 & 0.040 & $1.166-1.322$ & 1.2 \\
\hline $\begin{array}{l}\text { Servicios pagados } \\
\text { por empresa }\end{array}$ & 0.782 & 0.032 & $0.719-0.845$ & 0.8
\end{tabular}

Doble aseguramiento

\begin{tabular}{lcccc} 
IMSS & 11.987 & $0.69 \mid$ & $10.633-13.341$ & 12.0 \\
\hline No asegurados & 64.316 & 0.976 & $62.403-66.229$ & 64.3 \\
\hline ISSSTE & 15.173 & 0.726 & $13.750-16.596$ & 15.2 \\
\hline Pemex & 0.827 & 0.021 & $0.786-0.868$ & 0.8 \\
\hline Defensa-Marina & 0.450 & 0.149 & $0.158-0.742$ & 0.4 \\
\hline Otra inst. pública & 2.198 & 0.288 & $1.634-2.762$ & 2.2 \\
\hline $\begin{array}{l}\text { Servicios pagados } \\
\text { por empresa }\end{array}$ & 5.049 & 0.445 & $4.177-5.921$ & 5.0
\end{tabular}

Escolaridad y alfabetismo

\begin{tabular}{|c|c|c|c|c|}
\hline$<3$ años y no lee & 8.004 & 0.131 & $7.747-8.261$ & 8.0 \\
\hline$<3$ años y si lee & 9.110 & 0.143 & $8.830-9.390$ & 9.1 \\
\hline 3-6 primaria & 36.276 & 0.239 & $35.808-36.744$ & 36.3 \\
\hline Secundaria & 27.725 & 0.226 & $27.282-28.168$ & 27.7 \\
\hline Preparatoria & 9.884 & 0.151 & $9.588-10.180$ & 9.9 \\
\hline \multicolumn{5}{|l|}{ Profesional } \\
\hline o posgrado & 9.002 & 0.141 & $8.726-9.278$ & 9.0 \\
\hline
\end{tabular}

\section{(Continúa)}




\section{Cuadro IV}

\section{(Continuación)}

\begin{tabular}{|c|c|c|c|c|}
\hline \multirow[b]{3}{*}{ Variables } & \multicolumn{4}{|c|}{ SUDAAN y SPSS } \\
\hline & \multicolumn{3}{|c|}{ Corridas SUDAAN } & SPSS \\
\hline & $\%$ & S.E. & IC $95 \%$ & $\%$ \\
\hline \multicolumn{5}{|c|}{ Total de integrantes del hogar } \\
\hline I-2 miembros & 16.062 & 0.141 & $15.786-16.338$ & 16.1 \\
\hline 2-5 miembros & 37.339 & 0.181 & $36.984-37.694$ & 37.3 \\
\hline 6 y más miembros & 46.599 & 0.185 & $46.236-46.962$ & 46.6 \\
\hline
\end{tabular}

Población económicamente activa

\begin{tabular}{lllll} 
Activos & 50.028 & 0.258 & $49.522-50.534$ & 50.0 \\
\hline Inactivos & 49.972 & 0.258 & $49.466-50.478$ & 50.0
\end{tabular}

Población económicamente inactiva

Jubilados,

\begin{tabular}{lrrrc} 
pensionados & 7.016 & 0.185 & $6.653-7.379$ & 7.0 \\
\hline Estudiantes & 34.116 & 0.339 & $33.452-34.780$ & 34.1 \\
\hline Hogar & 58.860 & 0.354 & $58.166-59.554$ & 58.9
\end{tabular}

Edad por grupos quinquenales

\begin{tabular}{|c|c|c|c|c|}
\hline$<5$ Años & 2.005 & 0.061 & $1.885-2.125$ & 2.0 \\
\hline de 5 a 9 & 8.897 & 0.123 & $8.656-9.138$ & 8.9 \\
\hline de 10 a 14 & 11.742 & 0.140 & $11.468-12.016$ & 11.7 \\
\hline de 15 a 19 & 11.965 & 0.139 & II.693-12.237 & 12.0 \\
\hline de 20 a 24 & 11.341 & 0.137 & $11.072-11.610$ & 11.3 \\
\hline de 25 a 29 & 10.173 & 0.130 & $9.918-10.428$ & 10.2 \\
\hline de 30 a 34 & 7.805 & 0.116 & $7.578-8.032$ & 7.8 \\
\hline de 35 a 39 & 6.857 & 0.110 & $6.64 \mid-7.073$ & 6.9 \\
\hline de 40 a 44 & 6.000 & 0.102 & $5.800-6.200$ & 6.0 \\
\hline de 45 a 49 & 5.017 & 0.940 & $3.175-6.859$ & 5.0 \\
\hline de 50 a 54 & 4.177 & 0.086 & $4.008-4.346$ & 4.2 \\
\hline de 55 a 59 & 3.637 & 0.810 & $2.049-5.225$ & 3.6 \\
\hline de 60 a 64 & 2.735 & 0.070 & $2.598-2.872$ & 2.7 \\
\hline de 65 a 69 & 2.523 & 0.068 & $2.390-2.656$ & 2.5 \\
\hline de 70 a 74 & 1.701 & 0.056 & $\mid .591-1.811$ & 1.7 \\
\hline de 75 a 79 & 1.314 & 0.049 & $1.218-1.410$ & 1.3 \\
\hline de 80 a 84 & 0.786 & 0.039 & $0.710-0.862$ & 0.8 \\
\hline de 85 a 90 & 0.612 & 0.034 & $0.545-0.679$ & 0.6 \\
\hline de 90 y más & $0.7 \mid 4$ & 0.036 & $0.643-0.785$ & 0.7 \\
\hline
\end{tabular}

Pese a que la ENSA-II presenta algunas limitaciones inherentes a la técnica de la encuesta, las contribuciones de mayor relevancia, atribuibles a este trabajo, se orientan hacia una mejor comprensión del Sistema Nacional de Salud desde la perspectiva poblacional, en especial, hacia las condiciones y elementos que caracterizan la búsqueda y obtención de atención en los establecimientos que integran este sector en el ámbito nacional. Además constituye un valioso punto de partida en la generación de hipótesis para nuevos trabajos de investigación y evaluación, en especial, para la medición de cambios en los patrones y modos de utilización de la población mexicana antes y después de los cambios económicos del final de 1994; la encuesta serviría como medición basal.

Por todo lo anterior, es indudable que los resultados de la encuesta contribuirán -en la medida en que sean analizados y comparados- a ampliar el cuerpo de conocimientos en la materia para la mejor planeación y asignación de recursos en los servicios de salud en México, para promover su utilización eficiente y para reforzar las estrategias orientadas a fomentar la equidad del propio sistema.

La base de datos de esta encuesta esta a disposición de aquellos investigadores que requieran información sobre los patrones de uso de servicios de salud de la población mexicana. Para solicitarla es menester comunicarse con los autores de este trabajo al Instituto Nacional de Salud Pública, en la ciudad de Cuernavaca, Morelos, México.

\section{Agradecimientos}

Se agradece a todas las personas que colaboraron en el diseño de la ENSA-II, misma que fue producto del empeño institucional conjunto de la Dirección General de Epidemiología, la Dirección General de Estadística, Informática y Evaluación, el Centro de Estudios de Población y Salud, el Programa de Apoyo de Solidaridad en Salud a Población Abierta, el Instituto Nacional de Salud Pública y el Banco Mundial.

\section{Referencias}

I. Frenk J, Lozano R, Bobadilla JL. The epidemiologic transition in Latin America and the Caribbean:An update. Washigton, D.C.: Paper prepared for the Expert Meeting on New Findings and Developments in the Measurement of Mortality Levels, Patterns and Trends in Less Developed Countries, 1992:14-15.

2. Sepúlveda J, ed. Estrategias de cambio y líneas de acción. La reforma en salud. Mejorar la salud de los mexicanos. Organización y funcionamiento. México, D.F:: Cuadernos de Salud, Secretaría de Salud, 1994:33.

3. Ross D, Vaughan P. Health interview surveys in developing countries .A methodological review with recommendations for future surveys. Londres: EPC, 1986:4.

4. Fiedler J. A review of the literature on access and utilization of medical care with special emphasis on rural primary care. Soc Sci Med I98I; I5: 129 142. 
5. Andersen R. Health status indices and access to medical care. Am J Public Health 1978;68(5):458-463.

6.Aday LA, Andersen R.A framework for the study of access to medical care. Health Serv Res 1974;9(3):208-220.

7. Miners LA, Greene SB, Salber EJ, Schffler RM. Demand for medical care in a rural setting : Racial comparisons. Health Serv Res 1978; I3(3):26I275.

8. Mackinlay J. The help seeking behaviour of the poor. En: Kosa J, Zola I K, ed. Poverty and health, a sociological analysis.Cambridge (MA): Harvard University Press, 1976.

9. Newhouse J, Phelps CE. Policy options and the impact of national health insurance. J Calif Dent Asocc 1974;2(10):53-64.

10. Grossman M. On the content of health capital and the demand forhealth. J Polit Econ 1972;80:223-255.

I I. Hulka B, Wheat J. Patterns of utilization. Med Care 1985;23(5):438460 .

12. Davis K, Gold M, Makuc D. Access to health care for the poor: Does the gap remain. Annu Rev Public Health 1981;2:159-82.

13. MacKinlay J. Social network influences on morbid episodes and the career of help seeking behavior. En: Eisenberg L, Kleinman A, ed. The relevance of social science for medicine. Holanda: Reidel Publishing, 1981:78-89.

14. Leibowitz A, Friedman B. Familiy requests and the derived demand for health care. Econ Inq 1979; I7:133-139.

15. Aday LA, Andersen R. Insurance coverage and access: Implications for health policy. Health Serv Res 1978;13(4):369-377.

16. Donabedian A. La calidad de la atención médica. México, D.F.: La Prensa Médica, Mexicana 1974.

17. Donabedian A. La evaluación de la necesidad. En: Biblioteca de la Salud. Los espacios de la salud: aspectos fundamentales de la organización de la atención médica. México, D.F.: Fondo de Cultura Económica, 1988:71-83. I8. Frenk J. El concepto y medición de la accesibilidad. Salud Publica Mex 1985;27(5):438-453.

19. Research Triangle Institute. Survey Data Analysis Software; SUDAAN. Release 5.53, 1991 agosto.

20. SPSS Inc. Statistical Package for Social Sciences/PC+4.0, 1990

2I. Instituto Nacional de Estadística Geografía e Informática. IX Censo Nacional de Población y Vivienda. Aguascalientes: INEGI, I990. 